\title{
THE EFFECT OF RADIAL RADIATION TRANSPORT ON THE INTERPRETATION OF SATURATION PARAMETER MEASUREMENTS IN LASER SYSTEMS
}

\author{
G.J. ERNST \\ Twente University of Technology, Department of Applied Physics, Enschede, The Netherlands
}

Received 6 March 1978

\begin{abstract}
The influence of a spatial profile in the gain, which can be caused by saturation of the medium by the bearn, is studied on the amplification properties of the beam. It is found that the amplifying medium is about twice as efficiently depleted as can be expected on basis of a plane-wave approximation. This means that the saturation-parameter values as they have been deduced in the past are wrong by a factor two. The theoretical results are applied to $\mathrm{cw}^{\mathrm{CO}_{2}}$ measurements.
\end{abstract}

\section{Introduction}

A very important characteristic of any laser system is the total power output which can be generated. Because this output is determined by the saturation parameter of the laser system (and of course by the smallsignal gain), an accurate knowledge of its value, which can be obtained for instance from spontaneous-fluorescence measurements, is important. On the other hand determination of the saturation parameter from gain measurements can give an important contribution to the understanding of e.g. decay and relaxation processes in an atomic or molecular system. Finding the correct value of the saturation parameter is therefore a prerequisite.

In performing the calculations of the saturation parameter from gain versus input intensity measurements up till now a plane-wave approximation has been used (see e.g. Rigrod's paper [1]), i.e. the input beam, which usually has a fundamental mode structure, has been approximated by a plane wave propagating in the direction of the longitudinal axis of the amplifier having an effective surface determined by the width of the beam. Such an approximation, however, does not take inhomogeneous gain and refractive-index distributions into account. In this paper it will be shown that the gain profile, caused by spatial inhomogeneous saturation of the medium by the gaussian shaped beam, has a large impact on the interpretation of the gain measurements. In fact a wrong value for the saturation parameter will be obtained by a planewave approximation. Our analysis of the gain behaviour of an amplifier will solve a contradiction in literature.

\section{Theoretical analysis}

When the saturating beam and the laser amplifier have axial symmetry, as is usually the case, the complex propagation constant of the medium can be expanded in terms of the radial distance $r$ from the symmetry axis $z$, whereas the terms in odd power can be omitted. Because the intensity of the gaussian-shaped beam is highest near the axis, the constant and quadratic terms are the most important ones. Anyhow a treatment of the amplification where $k$ is given by

$$
\begin{aligned}
& k(r, z)=k_{0}(z)-\frac{1}{2} k_{2}(z) r^{2} \\
& \quad=\beta_{0}(z)+i \alpha_{0}(z)+\frac{1}{2}\left\{\beta_{2}(z) r^{2}+i \alpha_{2}(z) r^{2}\right\}
\end{aligned}
$$

will be an important improvement over the existing empty-space theory with plane-wave amplification. The theoretical basis for the amplification of a gaussian beam in a complex lens-like medium has already been developed in [2] and [3]. An extended survey can be found in [4]. It has been derived that the propagation of a gaussian beam throughout a complex lens-like medium is determined by the following two equations, 
where the same dimensionless notation has been used as in $[4]$ :

$P^{2}+\mathrm{d} P / \mathrm{d} \zeta+\eta^{2}=0$,

$\frac{1}{I} \frac{\mathrm{d} I}{\mathrm{~d} \zeta}=2\left(\alpha_{0} L-U\right)=2 \alpha_{0} L+\frac{1}{Q} \frac{\mathrm{d} Q}{\mathrm{~d} \zeta}+\frac{\alpha}{Q}$.

$P$ is the complex beam parameter defined by $P=U-$ $\mathrm{i} Q$, where $U=L / R$ and $Q=\lambda L / \pi w^{2} . L$ is the length of the active medium, $\zeta=z / L, \lambda$ is the wave length of the light beam, $R$ and $w$ are the radius of curvature and the width of the gaussian beam respectively. $\eta^{2}$ and $\alpha$ account for the profile in the complex refrac. tive index and are defined by

$\eta^{2}=\frac{k_{2}}{k_{0}} L^{2}=-(\beta+\mathrm{i} \alpha) \approx-\frac{\lambda}{2 \pi}\left(\beta_{2} L^{2}+\mathrm{i} \alpha_{2} L^{2}\right)$.

The intensity $I$ of the beam is the value at the axis $(r=0)$.

Eq. (2) determines the transformation of the parameters $R$ and $w$ of the gaussian beam when it propagates through the medium. It is identical with Kogelnik's $A B C D$ law. If the medium parameter $\eta^{2}$ is $\zeta$-independent, Kogelnik's law can be used with advantage be. cause the $A, B, C$ and $D$ values, which are the elements of the paraxial ray matrix, can be determined easily. However, since in an amplifier the intensity grows (for small input intensities the growth is even exponential), the medium parameter, being intensity-dependent, cannot be taken as constant over the length of the tube. This means that eqs. (2) and (3) have to be solved simultaneously and the changes of the beam properties (i.e. the beam width, the radius of curvature of the wave front and the intensity) can only be solved by numerical methods.

Eq. (3) determines the intensity growth of the beam when it propagates through the medium. The first term of the right-hand side describes the local conversion of inverted states into stimulated emission. This is the same term as found for a one-dimensional treatment with plane waves. The second term describes how the intensity is changed by a change in the width of the beam and is related to the continuity of propagating electromagnetic energy. In the absence of interaction the product of the beam cross-section and the intensity is constant along the axis. Therefore this term simply describes focussing or defocussing of the beam. The last term cannot be attributed to a simple effect like focussing. It described a contribution to the intensity at the axis induced by the three-dimen. sional character of the beam itself or by the spatially inhomogeneous gain distribution. Thus it describes a transport of radiation in radial direction. When $\alpha$ is positive, which means that the gain increases with the distance from the axis, it describes that the medium is more efficiently depleted than can be expected from plane-wave considerations.

\section{The gain characteristics of an amplifier}

As we have already pointed out in the preceding section, the growth of the intensity along the axis makes it necessary to solve eqs. (2) and (3) simultaneously. This can only be done by numerical methods. The easiest way to do this is to split up the amplifier into a large number of parts of equal length. Then the $\alpha_{0}, \alpha$ and $\beta$ values of any part can be calculated from the beam properties at the end of the former part. With these values of $\alpha_{0}, \alpha$ and $\beta$ taken as constant over the amplifier part the increase in intensity over that part can be determined by means of eq. (3) and the changes of the beam width and the radius of curvature of the wave front by means of Kogelnik's matrix-multiplication law. In this way the influence of the total amplifier on the beam properties can be calculated. We shall do this for both types of broaden. ing.

Homogeneously broadened medium. Al though it is possible to calculate the gain characteristics for very general cases, including refractive-index profiles due to the heat production in a gas discharge or dispersion effects, we shall treat only the simplest case where no profiles in the refractive index are present, i.e. $\beta=0$, which means that the frequency of the incoming beam coincides with the line centre and where no profile in the gain due to an inhomogeneous distribution of the small-signal gain or the saturation parameter is present. Thus a profile in the gain can only be caused by spatially inhomogeneous saturation of the medium by gaussian intensity distribution of the beam. In this way the effect of radial radiation transport can be seen most clearly and can be compared with the planewave calculations.

For the amplitude gain it then follows: 
$\alpha_{0}=\left(\frac{1}{2} \text { plane-wave gain }\right)_{r=0}=\frac{\frac{1}{2} G_{0}}{1+I / I_{0}}$,

where $G_{0}$ is the small-signal intensity gain per unit length at the line centre. $I_{0}$ is the saturation parameter at the line centre and $I$ is the intensity of the beam at the axis. Since the intensity of the beam is given by

$I(r)=I \exp \left(-2 r^{2} / w^{2}\right)$

it follows for $\alpha_{2}$ :

$\alpha_{2}=\left(\frac{1}{2} \frac{\mathrm{d}^{2}}{\mathrm{~d} r^{2}} \text { plane-wave gain }\right)_{r=0}=\frac{G_{0}\left(2 / w^{2}\right)\left(I / I_{0}\right)}{\left(1+I / I_{0}\right)^{2}}$

where $w$ is the width of the gaussian beam. Thus for $\alpha$ follows:

$\alpha=\frac{\lambda}{2 \pi} \alpha_{2} L^{2}=\frac{G_{0} L Q I / I_{0}}{\left(1+I / I_{0}\right)^{2}}$.

Substituting eqs. (5) and (7) into (3) gives:

$\frac{1}{I} \frac{\mathrm{d} I}{\mathrm{~d} \zeta}=\frac{G_{0} L}{1+I / I_{0}}\left(1+\frac{I / I_{0}}{1+I / I_{0}}\right)+\frac{1}{Q} \frac{\mathrm{d} Q}{\mathrm{~d} \zeta}$.

In this equation the effect of the radial radiation transport on the gain can clearly be seen. For small values of the gain $G_{0}$ the beam width will not be changed very much, so that the last term on the right-hand side will be zero. When the input intensity is high compared to the saturation parameter, the radial radiation transport contributes as much to the total gain as the direct conversion of inverted states. This means that the medium is twice as efficiently depleted as can be expected from plane-wave considerations. For higher values of the gain this conclusion will be essentially the same.

Because

$\frac{1}{I} \frac{\mathrm{d} I}{\mathrm{~d} \zeta}-\frac{1}{Q} \frac{\mathrm{d} Q}{\mathrm{~d} \zeta}=\frac{1}{P} \frac{\mathrm{d} P}{\mathrm{~d} \zeta}$,

where $P$ is the power of the beam, eq. (8) gives directly the power gain of the beam. Although for many cases expression (8) will be sufficiently accurate for calculating the power gain (the intensity $I$ can be found by dividing the power through an average value of the beam area), we have performed the calculations of the intensity gain by solving eq. (8) together with eq. (2) according to the procedure outlined in the beginning of this section. The results are plotted in fig. 1.

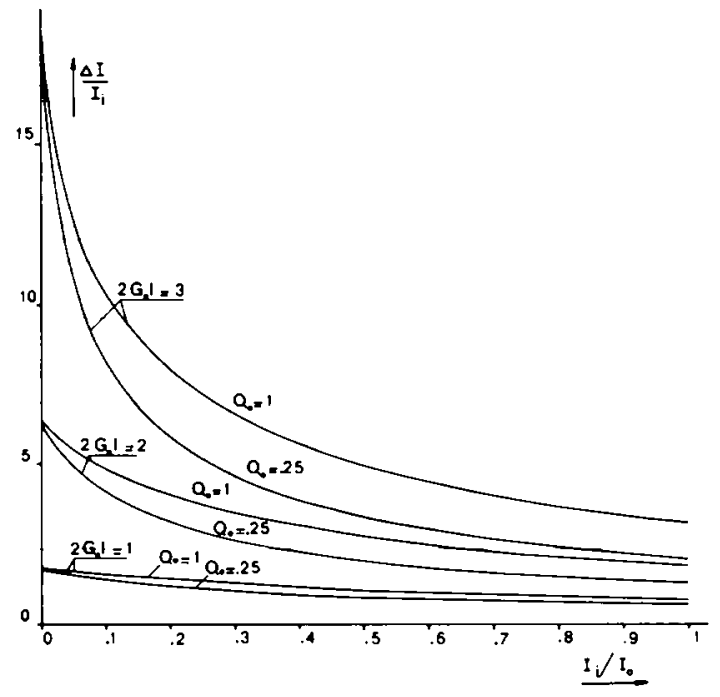

Fig. 1. The intensity gain at the centre of the axis plotted for a homogeneously broadened medium as a function of the normalized input intensity. The total small-signal gain $2 G_{0} L$ has been used as a parameter. $Q_{0}$ is the value of $\lambda L / \pi w_{0}^{2}$ for very small input intensities halfway along the amplifier.

Because the result of the calculations depends on the beam width and radius of curvature of the incoming beam at the entrance plane, we have to specify those values. The values have been chosen in such a way that in empty space the beam waist would be half-way along the amplifier and has the value $w_{0}$. The parameter $Q_{0}=\lambda L / \pi w_{0}^{2}$ in the figure has been used for characterizing this situation. The total length of the amplifier has been chosen as $2 L$, so that the total small-signal gain of the amplifier is $2 G_{0} L$.

From a theoretical point of view the determination of the saturation parameter from intensity-gain measurements is preferable to a determination from powergain measurements because influences from higherorder terms than the constant and quadratic ones of the gain mainly affect the power gain, whereas the intensity gain at the axis of the tube will only slightly be altered, especially for $Q_{0}$ values smaller than 1 . However, in practice one often has to determine the power gain as a function of the incident power, simply because measurements of the intensity gain are not accurate enough. Because the beam width at the exit of the amplifier usually is very different from the empty-space value, the power gain behaves much more differently than the intensity gain. The results 


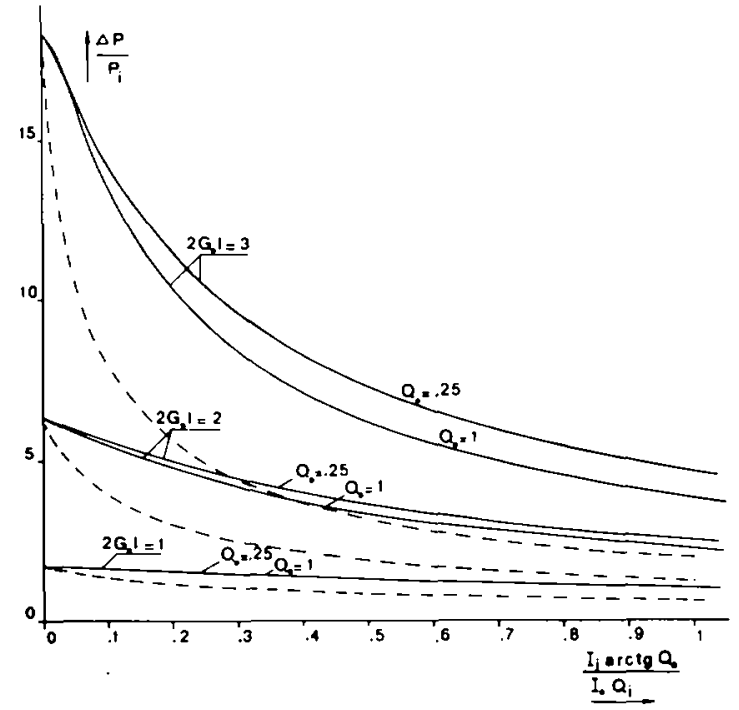

Fig. 2. The power gain plotted for a homogeneously broadened medium as a function of an averaged input intensity. Again $2 G_{0} L$ and $Q_{0}$ are used as a parameter. The dashed lines show the gain for the case that the laser beam has been approximated by a plane wave.

for the power gain can be seen in fig. 2. At the horizontal axis we have plotted the quantity

$\frac{\bar{I}_{\mathrm{i}}}{I_{0}}=\frac{I_{\mathrm{i}}}{I_{0}} \frac{\operatorname{arctg} Q_{0}}{Q_{\mathrm{i}}}$,

where $Q_{\mathrm{i}}$ is the $Q$ value at the entrance of the amplifier given by

$Q_{\mathrm{i}}=Q_{0} /\left(1+Q_{0}^{2}\right)$,

and where $I_{\mathrm{i}}$ is the entrance value of the intensity at the axis of the beam. The $\operatorname{arctg} Q_{0}$ value appears in the expression because we averaged the $Q$ value in vacuum over the length of the amplifier:

$\bar{Q}=\frac{1}{2 L} \int_{-L}^{L} Q \mathrm{~d} z=\frac{1}{L} \int_{0}^{L} \frac{Q_{0}}{1+\left(Q_{0} z / L\right)^{2}} \mathrm{~d} z=\operatorname{arctg} Q_{0}$.

Hence the quantity $\bar{I}_{\mathrm{i}} / I_{0}$ is some kind of averaged input intensity. We have chosen this quantity, which is of course somewhat arbitrary, in order to compare our calculations directly with the experimental results reported in literature.

We have also compared our calculations based on the three-dimensional model with the one-dimensional treatment which is normally used by others (see [1]). The results are also plotted in fig. 2 (dashed lines). At the horizontal axis the input intensity of the plane wave divided by the saturation parameter is plotted. One can easily see the big difference between the plane wave model and our model. The conclusion from this is that values of the saturation parameter as they have always been obtained in the past by fitting the measurements to calculations with the plane-wave model are wrong. In the next section we shall apply our results to the excellent gain measurements published by Christensen, Freed and Haus [5]. It will prove the value of our theory.

Inhomogeneously broadened medium. Also in this case we treat only the simplest case, where no refractive-index profiles are present (i.e. $\beta=0$ ) and where the small-signal gain and saturation parameter have no space-dependence. Thus the profile in the gain can only be caused by spatially inhomogeneous saturation of the medium by the gaussian intensity distribution of the beam.

For the amplitude gain at the line centre at the axis of. the tube it now follows for an inhomogeneously broadened transition:

$\alpha_{0}=\frac{\frac{1}{2} G_{0}}{\left(1+I / I_{0}\right)^{1 / 2}}$

and:

$\alpha_{2}=\frac{\left(G_{0} / w^{2}\right)\left(I / I_{0}\right)}{\left(1+I / I_{0}\right)^{3 / 2}}$,

so that

$\alpha=\frac{1}{2} \frac{Q G_{0} L I / I_{0}}{\left(1+I / I_{0}\right)^{3 / 2}}$.

Substituting eqs. (13) and (15) into eq. (3) gives:

$\frac{1}{I} \frac{\mathrm{d} I}{\mathrm{~d} \zeta}=\frac{G_{0} L}{\left(1+I / I_{0}\right)^{1 / 2}}\left(1+\frac{1}{2} \frac{I / I_{0}}{1+I / I_{0}}\right)+\frac{1}{Q} \frac{\mathrm{d} Q}{\mathrm{~d} \zeta}$.

From eq. (16) it can be seen that in the case of an inhomogeneously broadened medium the influence of the radial radiation transport is less than for homogeneous broadening. For strong saturation its contribu. tion to the intensity increase is about half the amount due to direct conversion of inverted states. Although also in this case eq. (16) can give the power increase to a good approximation, we have calculated the in- 


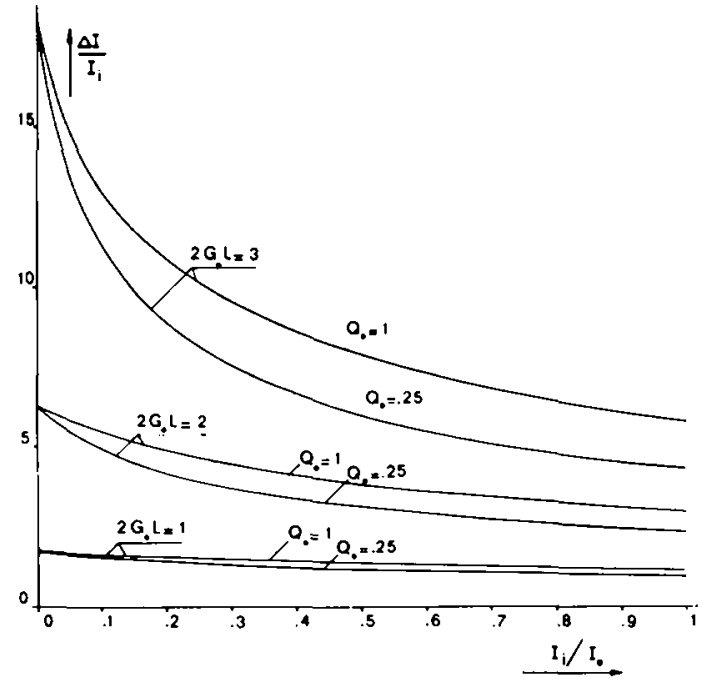

Fig. 3. The intensity gain at the centre of the axis plotted for an inhomogeneously broadened medium as a function of the normalized input intensity. The total small-signal gain $2 G_{0} L$ has been used as a parameter. $Q_{0}$ is the dimensionless quantity $\lambda L / \pi w_{0}^{2}$ for very small input intensities half-way along the amplifier.

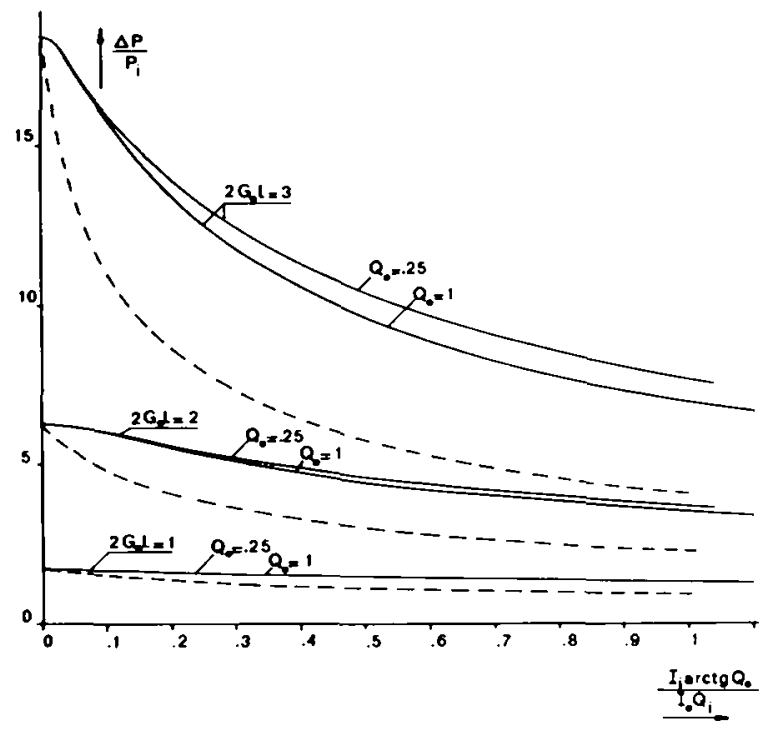

Fig. 4. The power gain plotted for an inhomogeneously broadened medium as a function of an averaged input intensity. Again $2 G_{0} L$ and $Q_{0}$ are used as a parameter. The dashed lines show the gain for the case when the laser beam has been approximated by a plane wave. crease in intensity over a passage through the active medium in the same way as for the homogeneous case. The results are plotted in fig. 3 . The power gain is plotted in fig. 4 . For both figures we used the same parameter definition and the same quantities at the axis as for the homogeneously broadened transition. It can be seen from these figures that also in this case there is a big difference between our model and the plane-wave model (dotted lines).

\section{Application to the $\mathrm{cw} \mathrm{CO}_{2}$ system}

In order to prove that our model is the right one we can make gain measurements and compare the deduced saturation parameter with the saturation parameter that can be found from lifetime measurements of the laser level. However, in literature excellent gain measurements have been published. As an example we shall look at the measurements published by Christensen, Freed and Haus [5]. They measured the power gain of an amplifier having a length of $70 \mathrm{~cm}$ and deduced the saturation parameter from their measurements using the plane-wave model. By doing this for several beam diameters they found a beam diameter-dependent saturation parameter. This beamdependence must be attributed to the diffusion of the excited $\mathrm{CO}_{2}$ molecules as a result of spatial differences of the inversion density. Correction for this diffusion (this can be done e.g. by extrapolating the values for the saturation parameter at different beam diameters to the value for a very large beam diameter) leads to a value of about $18 \mathrm{~W} / \mathrm{cm}^{2}$ for $I_{0}$.

We can now estimate the saturation parameter from lifetime measurements. The $\mathrm{CO}_{2}$ laser has a homogeneously broadened transition. The expression for the saturation parameter at the line centre for small excitation rates is given by (see e.g. [4]):

$$
\begin{aligned}
I_{0} & =\frac{\pi \Delta \nu}{2 B_{21}} \frac{A_{1} A_{2}}{A_{1}+\left(g_{2} / g_{1}\right)\left(A_{2}-A_{21}\right)} \\
& =\frac{4 \pi^{2} h \nu \Delta \nu}{A_{21} \lambda^{2}\left\{1 / A_{2}+\left(g_{2} / g_{1}\right)\left(1 / A_{1}-A_{21} / A_{1} A_{2}\right)\right\}}
\end{aligned}
$$

$A_{2}$ and $A_{1}$ are the decay rates of the upper and lower laser levels respectively; $B_{21}$ and $A_{21}$ are the coefficient for stimulated emission and the decay rate between upper and lower laser levels respectively. $\Delta \nu$ is 
the line width of the transition.

Substituting the following values for their experimental circumstances:

$$
\begin{array}{ll}
1 / A_{21}=5.2 \mathrm{~s}([6,7]), & 1 / A_{2}=0.82 \mathrm{~ms}([8]), \\
1 / A_{1} \ll 1 / A_{2}, & \Delta \nu=86 \mathrm{MHz}([9]),
\end{array}
$$

we find a value of $0.34 \mathrm{~W} / \mathrm{cm}^{2}$ for the saturation parameter. This value is for only one rotational transition. However, in a $\mathrm{CO}_{2}$ laser all rotational transitions are very strongly coupled by cross relaxation. This means that not only the inversion of one rotationvibration transition determines the saturation parameter, but the total inversion of all rotational transitions. Under excitation conditions the $J=20$ rotational transition is the strongest one, which means that the population is highest for that $J$ value. Taking the sum over all rotational transitions it appears that the saturation parameter will be about 14 times the value of one rotational transition. Hence the saturation parameter can be expected to be about $4.7 \mathrm{~W} / \mathrm{cm}^{2}$ and there is a discrepancy between the value derived from lifetime measurements and that obtained from gain measurements analyzed by the plane-wave amplification model. The existence of this discrepancy has been pointed out by Christensen et al. but an explanation could not be given. We have calculated the gain behaviour according to our model for their experimen. tal circumstances (viz. $2 G_{0} L=0.41$ ). The results are plotted in fig. 5 . The curves for different $Q_{0}$ values cannot be distinguished at this value of $2 G_{0} L$. The lower curve in this figure gives the gain behaviour for the plane-wave amplification.

The most common way of determining the saturation parameter (also used by Christensen et al.) is to draw the tangent to the gain curve at very low input intensities. The intersection of this tangent with the $\Delta P / P=0$ line determines the saturation parameter. For the plane-wave model this can easily be verified by deriving the quantity

$\left(\partial I_{\mathrm{u}} / I_{\mathrm{i}}\right) / \partial I_{\mathrm{i}}$

in the point $I_{\mathrm{i}}=0$, where $I_{\mathrm{i}}$ is the intensity of the incoming beam and $I_{\mathrm{u}}$ is the intensity after amplification.

One finds for this quantity:

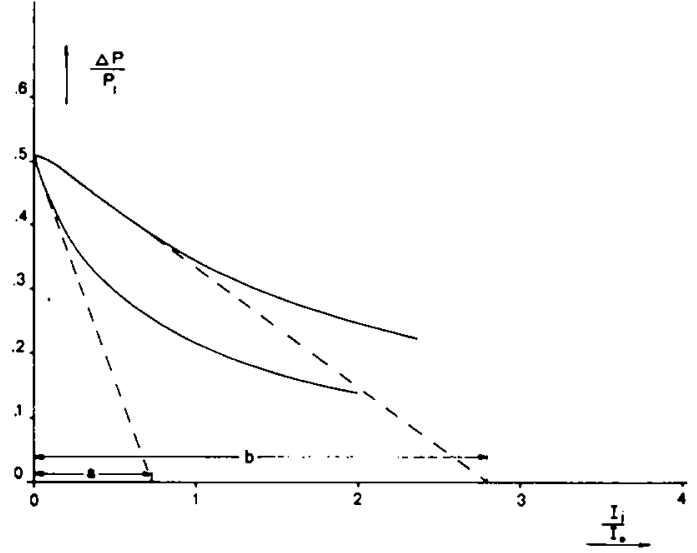

Fig. 5. The power gain plotted for a homogeneously broadened medium. The small-signal gain is $2 G_{0} L=0.41$. The lower curve shows the calculations for a plane-wave approximation. The dashed lines are the tangent lines to the curve for small input intensities.

$\operatorname{tg} \phi=\left(-\frac{\partial I_{\mathrm{u}} / I_{\mathrm{i}}}{\partial I_{\mathrm{i}}}\right)_{I_{\mathrm{i}}=0}=\frac{\mathrm{e}^{2 G_{0} L}\left(\mathrm{e}^{2 G_{0} L}-1\right)}{I_{0}}$,

where $2 G_{0} L$ is again the total amplification in the amplifier of length $2 L$. It can be seen from fig. 5 that according to our model $\operatorname{tg} \phi$ is $a / b$ times smaller. Hence when the tangent and the $\Delta P / P=0$ line intersect at the measured intensity of $I_{\mathrm{m}} \mathrm{W} / \mathrm{cm}^{2}$, the use of the plane-wave model enables us to calculate the saturation parameter:

$\operatorname{tg} \phi=\frac{\mathrm{e}^{2 G_{0} L}-1}{I_{\mathrm{m}}}=\frac{\mathrm{e}^{2 G_{0} L}\left(\mathrm{e}^{2 G_{0} L}-1\right)}{I_{0}}$,

or:

$I_{0}=I_{\mathrm{m}} \mathrm{e}^{2 G_{0} L}$

In the same way we find with our model:

$I_{0}=(a / b) I_{\mathrm{m}} \mathrm{e}^{2 G_{0} L}$

From the figure it can be seen that for $2 G_{0} L=0.41$ the $a / b$ value is about 0.28 . The saturation parameter deduced according to our model is then $I_{0}=0.28 \mathrm{X}$ $18 \mathrm{~W} / \mathrm{cm}^{2}=5 \mathrm{~W} / \mathrm{cm}^{2}$ and is in excellent agreement with the lifetime measurements.

So we find a factor of almost 4 between the saturation parameter derived by the two different models. Looking at eq. (8) one would expect the factor to be about 2 . This difference is due to the fact that at the 
horizontal axis of the gain characteristics the quantity $P_{\mathrm{i}} / \pi r^{2}$ is plotted, according to Christensen et al. and many others; $\bar{r}$ is the average over the length of the tube of the squared $1 / \mathrm{e}$ radius of the intensity of the beam. However, for a gaussian shaped beam $P_{\mathrm{i}} / \pi r^{2}$ equals $P_{\mathrm{i}} / \frac{1}{2} \pi w^{2}$, where $w$ is the beam width, which is just equal to the central intensity value of the beam. It would have been better to plot instead some kind of an effective value of the intensity over the beam area, which then can be averaged over the length of the tube. When the quantity $P_{\mathrm{i}} / \pi \overline{w^{2}}$ is plotted, the discrepancy between the plane-wave model and our three-dimensional model will be about a factor 2 .

\section{Conclusions}

It may be concluded that deducing the saturation parameter from gain measurements by a plane-wave analysis is not correct. A wrong value will be obtained. The radial radiation transport due to a spatial profile in the gain as will be caused by saturation has to be included in order to find the right value. A quadratic approximation has been used for calculating the right value of the saturation parameter. Application of the theory to the gain measurements of a $\mathrm{cw} \mathrm{CO}_{2}$ laser shows that this approximation is sufficiently accurate.

\section{References}

[1] W.W. Rigrod, J. Appl. Phys. 34 (1963) 2602.

(2) G.J. Ernst and W.J. Witteman, IEEE J. Quant. Electron. QE-10 (1974) 37.

[3] W.J. Witteman and G.J. Ernst, IEEE J. Quant. Electron. QE-11 (1975) 198.

[4] G.J. Ernst, Ph. D. Thesis, Twente University of Technology, Enschede, The Netherlands, 1977.

[5] C.P. Christensen, C. Freed and H. Haus, IEEE J. Quant. Electron. QE-5 (1969) 276.

[6] E.T. Gerry and D.A. Leonard, Appl. Phys. Letters 8 (1966) 227.

[7] D.W. Ducsik, B.S. Thesis, Massachusetts Institute of Technology, Cambridge, Mass., USA, 1968.

[8] W.A. Rosser, A.D. Wood and E.T. Gerry, Paper 7G-5, Qu. El. Conf., Miami, Florida, May 1968.

[9] T.J. Bridges, H.A. Haus and P.W. Hoff, IEEE J. Quant. Electron. QE-4 (1968) 777. 